\title{
The Caenorhabditis elegans gene lin-10 is broadly expressed while required specifically for the determination of vulval cell fates
}

\author{
Stuart K. Kim ${ }^{1-3}$ and H. Robert Horvitz ${ }^{1}$ \\ ${ }^{1}$ Howard Hughes Medical Institute, Department of Biology, Massachusetts Institute of Technology, Cambridge, \\ Massachusetts 02139, USA; ${ }^{2}$ Department of Developmental Biology, Stanford Medical School, Stanford University, Stanford, \\ California 94305 USA
}

\begin{abstract}
As a first step in a molecular dissection of the pathway controlling the determination of vulval cell fates in Caenorhabditis elegans, we have analyzed the gene lin-10. We show that loss-of-function mutations in this gene specifically prevent the induction of vulval cell lineages and result instead in the expression of hypodermal cell lineages. We isolated a transposon-insertion allele of lin-10 and used it to clone a genomic region that contains the lin-10 locus. The location of lin-10 within this region was determined by identifying a transcript affected by three independent lin-10 mutations and by delimiting the minimal segment of DNA sufficient to rescue the lin-10 mutant phenotype in germ line transformation experiments. The predicted lin-10 protein sequence is not similar to sequences in current data bases, suggesting that lin-10 defines a novel class of gene involved in the specification of cell fates. Although our genetic studies indicate that lin-10 is required specifically for the determination of vulval cell fates, lin-10 transcripts are present in cells other than vulval precursor cells. This result suggests that lin-10 may have a general but redundant role in development, functioning in diverse cell lineages to control cell fates. Alternatively, lin-10 may function specifically in vulval development, in which case lin-10 activity could be regulated at a post-transcriptional level or could have biological consequences only in conjunction with the products of other genes.
\end{abstract}

[Key Words: lin-10 gene; vulval cell lineage; Caenorhabditis elegans]

Received November 11, 1989; revised version accepted December 14, 1989.

To understand how genes control cell fates during development, we are studying the cell lineages that give rise to the vulva of the Caenorhabditis elegans hermaphrodite. Vulval development involves the induction of vulval cell lineages from a set of six equipotential precursor cells (P3.p; P4.p, P5.p, P6.p, P7.p, and P8.p) by a regulatory cell (the gonadal anchor cell); (Fig. 1); (Sulston and Horvitz 1977; Sulston and White 1980; Kimble 1981; Sternberg and Horvitz 1986). Each of the six precursor cells, collectively called Pn.p cells, is capable of expressing one of three types of lineages, referred to as $1^{\circ}, 2^{\circ}$, and $3^{\circ}$. The $1^{\circ}$ and $2^{\circ}$ cell lineages generate cells that form the vulva, whereas the $3^{\circ}$ cell lineage generates cells that form part of the ventral hypodermis.

Which of these three alternative fates a given Pn.p cell will express is determined primarily by the proximity of the Pn.p cell to the anchor cell (Sulston and White 1980; Kimble 1981; Sternberg and Horvitz 1986). The Pn.p cell closest to the anchor cell (P6.p) expresses the $1^{\circ}$ cell lin-

${ }^{3}$ Corresponding author. eage, the two adjacent Pn.p cells (P5.p and P7.p) express the $2^{\circ}$ cell lineage, and Pn.p cells located farther from the anchor cell (P3.p, P4.p, and P8.p) express the $3^{\circ}$ cell lineage. It has been proposed that the anchor cell is the source for a graded inductive signal, that the $1^{\circ}$ and $2^{\circ}$ lineages are induced by a strong or medium anchor cell signal, respectively, and that the $3^{\circ}$ cell lineage represents the ground-state lineage that is expressed in the absence of an anchor cell signal (Sternberg and Horvitz 1986). In addition to communicating with the anchor cell, Pn.p cells communicate among themselves: A Pn.p cell that expresses a $1^{\circ}$ cell lineage inhibits its neighbors from also expressing a $1^{\circ}$ cell lineage, helping to ensure that the wild-type $2^{\circ}-1^{\circ}-2^{\circ}$ pattern of vulval cell lineages is expressed (Sternberg 1988).

An extensive genetic analysis of vulval development has identified at least 17 genes, referred to as vulval determination genes, that are involved in specifying which of the three cell lineages a particular Pn.p cell will express /Greenwald et al. 1983; Ferguson and Horvitz 1985; Ferguson et al. 1987; G. Beitel, pers. comm.; S. 


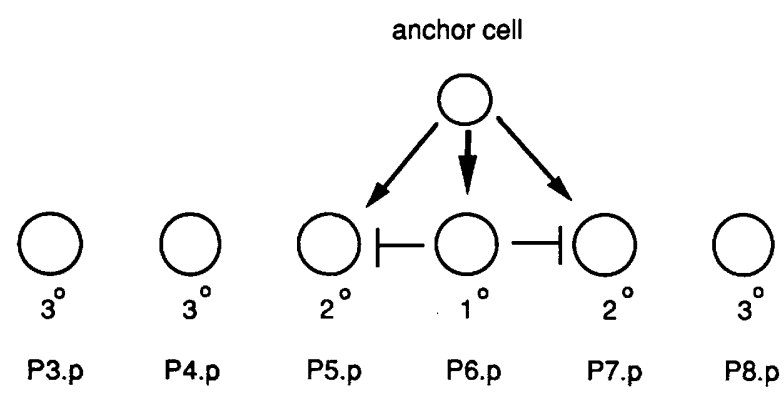

Figure 1. Model for the determination of Pn.p cell fates. Arrows indicate inductive interactions between Pn.p cells and the anchor cell; bars indicate communication between Pn.p cells. (For explanation, see text).

Kim, unpubl.). Mutations in these genes are homeotic in that they cause certain Pn.p cells to express the lineages normally expressed by other Pn.p cells. Specifically, mutations in 10 of these genes, including lin-15, cause all six Pn.p cells to adopt induced $\left(1^{\circ}\right.$ or $\left.2^{\circ}\right)$ cell lineages. This type of lineage transformation results in a multivulva phenotype, since the extra vulval cells appear as ectopic pseudovulvae on the ventral surface of the animal. Mutations in five genes, including lin-2 and lin-10, prevent the induction of vulval cell lineages, such that all six Pn.p cells adopt $3^{\circ}$ cell lineages. This transformation results in a vulvaless phenotype, because no vulval cells are formed. One gene, lin-31, has a mutant phenotype in which the determination of Pn.p cell fate may be deregulated; individual Pn.p cells appear to adopt randomly either a $1^{\circ}, 2^{\circ}$, or $3^{\circ}$ cell lineage (P. Sternberg, pers. comm.; S. Kim, unpubl.). Finally, lin-12 specifies whether a Pn.p cell will express a $2^{\circ}$ or non- $2^{\circ}$ cell lineage; Pn.p cells adopt $2^{\circ}$ cell lineages in lin-12 gain-offunction mutants and non $-2^{\circ}\left(1^{\circ}\right.$ or $\left.3^{\circ}\right)$ cell lineages in lin-12 loss-of-function mutants. These genes define two regulatory pathways that together specify the three Pn.p cell fates, because the genes in the multivulva/vulvaless class determine whether a Pn.p cell will express an induced or uninduced cell lineage, and lin-12 specifies whether an induced lineage is $1^{\circ}$ or $2^{\circ}$ (Sternberg and Horvitz 1989).

Laser ablation and genetic epistasis experiments have established that few or none of the vulval determination genes function solely in the anchor cell, which suggests that these genes function in Pn.p cells (Ferguson et al. 1987). Furthermore, genetic mosaic experiments have revealed that $l i n-12$ acts in a cell-autonomous manner in specifying ventral uterine cell fates (Seydoux et al. 1989), which suggests that this gene may also act in a cell-autonomous manner in specifying Pn.p cell fates. Together, these results lead to the hypothesis that most or all of the vulval determination genes function in Pn.p cells and that these genes define a signaling pathway for the response of Pn.p cells to extracellular signals. This pathway may include genes involved in every step of cellular determination, from reception of the anchor cell signal at the cell surface, through transduction of the signal in the cytoplasm, to the induction of lineage-specific gene expression in the nucleus.
We have begun a molecular analysis of this signaling pathway. As a first step toward the cloning of these vulval determination genes, we have isolated putative transposon-insertion mutations in five of these loci. We have analyzed one of these genes, lin-10, in detail, both genetically and molecularly, and show that the predicted lin-10 protein sequence is not similar to sequences in current data bases. This result indicates that the specific mechanism used by lin-10 to specify cell fate may be different from that of regulatory genes that have been characterized previously. We also show that 1 in -10 is expressed in cells other than Pn.p cells, even though the phenotype resulting from the elimination of lin-10 gene activity appears to be specific for Pn.p cells. One possibility is that there is an activity that is redundant with lin-10 in cells other than Pn.p cells. Another possibility is that lin-10 function is dependent on vulval determination genes that are active only in Pn.p cells.

\section{Results}

Transposon-insertion mutagenesis of genes that specify Pn.p cell fates

In certain C. elegans mutator strains, spontaneous mutations arise at a high frequency, often as a result of the insertion of transposons such as Tc1 (Collins et al. 1987) and Tc3 (Finney et al. 1988; Collins et al. 1989). Genes with transposon-insertion alleles can be cloned directly using available transposon probes (Greenwald 1985; Moerman et al. 1986). To identify transposon-insertion mutations in genes that control Pn.p cell determination, we screened the mutator strain TR679 (Collins et al. 1987) for multivulva and vulvaless mutants. We isolated 16 mutations, which are alleles of 5 previously identified genes: lin-2 (three alleles), lin-10 (two alleles), lin-12 (one allele), lin-15 (three alleles), and lin-31 (nine alleles) (see Experimental procedures Horvitz and Sulston 1980; Ferguson et al. 1987)

lin-12 has been cloned previously (Greenwald 1985), and Southern blotting experiments indicate that the mutator-induced allele isolated in this study, $n 1295$, is a Tc1 insertion (S. Kim, unpubl.). The other 15 mutations may also be caused by transposon insertions, enabling us to clone these four vulval determination genes in a straightforward manner.

\section{lin-10 may be required only for the determination of Pn.p cell fates}

We have begun our molecular studies of genes in the vulval determination pathway by cloning and analyzing lin-10. Mutations in lin-10 transform vulval cell lineages $\left(1^{\circ}\right.$ and $\left.2^{\circ}\right)$ into hypodermal cell lineages $\left(3^{\circ}\right)$, resulting in a vulvaless phenotype. As shown below, lin-10 gene activity appears to be required exclusively for the determination of Pn.p cell fates, because mutations that eliminate 1 in-10 activity affect only these cells. In contrast, mutations in almost all other vulval determination genes (including lin-15 and lin-31) have pleiotropic ef- 
fects, many of which are likely to reflect lineage transformations of other cells /Greenwald et al. 1983; Ferguson and Horvitz 1985; P. Sternberg; M. Herman; both pers. comm.; S. Kim, unpubl.).

Our assertion that 1 in-10 activity is required specifically to determine Pn.p cell fates is based on several lines of evidence. First, all 19 existing lin-10 alleles (of which 16 were isolated in this study; see Experimental procedures) result in a similar vulvaless phenotype but otherwise do not appear to affect behavior or morphology (see Experimental procedures; Horvitz and Sulston 1980; Ferguson and Horvitz 1985). Second, the phenotype of lin-10(e1439)/deficiency heterozygotes is vulvaless (Ferguson and Horvitz 1985), showing that a twofold reduction in gene dose does not result in a more severe phenotype. Third, 8 of the 16 new lin-10 alleles were isolated on the basis of their failure to complement the 1 in-10 allele $e 1439$ (see Experimental procedures). Because 1in-10(e1439)/deficiency animals are vulvaless, screens of this sort can identify null alleles even if they result in recessive lethality. A significant fraction of mutations isolated in this way should eliminate lin-10 gene activity. All eight alleles resulted in a recessive vulvaless phenotype without other obvious defects. Fourth, these new alleles were isolated at a frequency $\left(1.4 \times 10^{-4}\right.$ per mutagenized haploid genome $)$ that is close to the average frequency for the generation of null mutations $\left(5 \times 10^{-4}\right.$ per mutagenized genome $)$, using EMS mutagenesis (Brenner 1974; Meneely and Herman 1979; Greenwald and Horvitz 1980). Fifth, one of the new lin-10 alleles tested, $n 1638$, is suppressible by the amber suppressor tRNA mutation sup-5(e1464) (see Experimental procedures); amber mutations usually result in premature termination of the protein and, in many cases, in the generation of a nonfunctional gene product. Taken together, these results suggest that lin-10 gene function may be required primarily, if not solely, for the determination of the fates of only three cells, P5.p, P6.p,

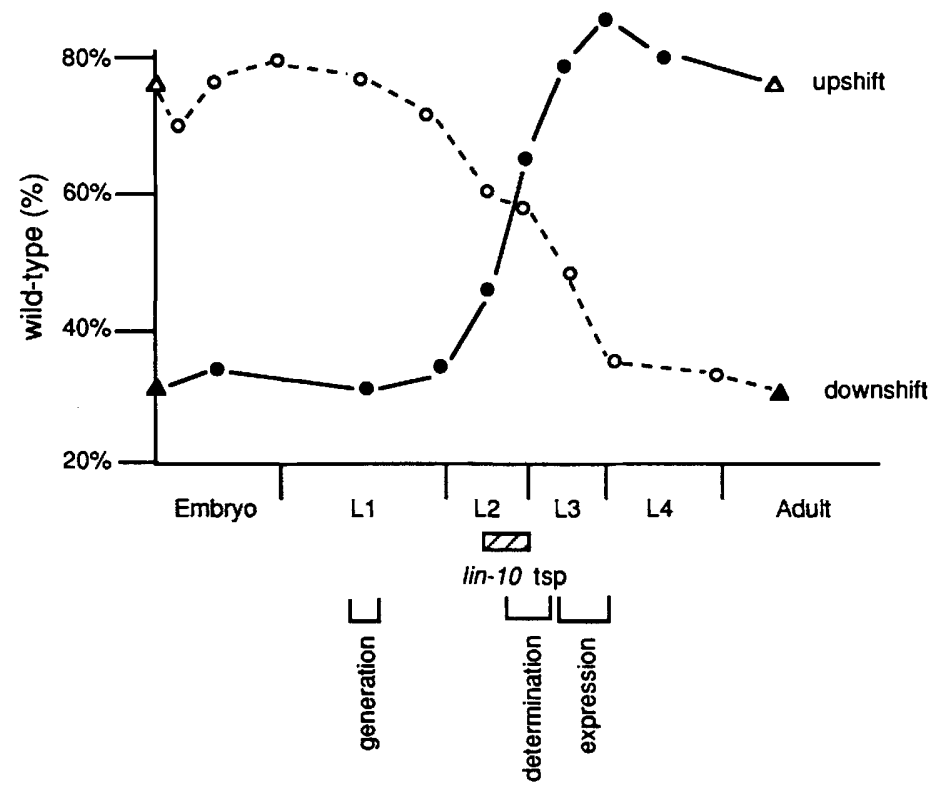

and P7.p. However, it remains possible that lin-10 mutations cause subtle defects that have not yet been detected.

The lin-10 temperature-sensitive period coincides with the time of Pn.p cell determination

One of the newly identified lin-10 alleles, $n 1509$, is temperature-sensitive: approximately $30 \%$ of $n 1509$ animals grown at $15^{\circ} \mathrm{C}$ are vulvaless, whereas about $75 \%$ of the animals grown at $25^{\circ} \mathrm{C}$ are vulvaless. We have used $n 1509$ to determine the temperature-sensitive period (TSP) for the lin-10 vulvaless phenotype. n1509 animals were shifted from one temperature to the other $\left(15-25^{\circ} \mathrm{C}\right.$ or vice versa) at various stages during development and later scored for their vulval phenotypes. As shown in Figure 2, the 1509 TSP extends from late in the second larval period to early in the third larval period. Presumably, either the synthesis or the activity of the lin-10(n1509) gene product is temperature-sensitive. In either case, the TSP is likely to correspond closely to the time during which lin-10 functions; lin-10 must function after the onset of the TSP in the late second larval stage, but before the beginning of Pn.p cell divisions in the third larval stage (at which time the mutant phenotype becomes apparent).

The time of $l i n-10$ function is indistinguishable from the time during which Pn.p cell fates are being determined (Sternberg and Horvitz 1986), which supports the idea that $l i n-10$ plays a direct role in determining Pn.p cell fates in response to the inducing signal from the anchor cell. Furthermore, lin-10 is likely to interact with some of the other vulval determination genes, such as lin-12, lin-13, lin-15, and let-23. These genes also have TSPs that overlap with the time of Pn.p cell determination and thus are likely to function at essentially the same time as lin-10 (Ferguson et al. 1987).

Figure 2. TSP for lin-10(n1509ts). Staged lin-10(n1509ts) animals were shifted from $15-25^{\circ} \mathrm{C}(0)$ or $25^{\circ} \mathrm{C}$ to $15^{\circ} \mathrm{C}(\mathrm{O})$ at the indicated times (see Experimental procedures). About 200-400 animals were scored for each point, and the percent that were non-vulvaless is shown. The percent of nonvulvaless animals grown continuously at $15^{\circ} \mathrm{C}(\Delta)$ and $25^{\circ} \mathrm{C}$ (A) are also shown. The lin-10 TSP is shown as a hatched box and indicates the minimal period during which the gene product must be active to generate a wild-type phenotype. The beginning of the TSP is defined as the earliest point at which a downshift $(O)$ results in a decrease in the percentage of phenotypically wild-type animals (Suzuki 1970). The end of the TSP is defined as the latest point at which an upshift (O) results in a decrease in the percentage of animals with a wild-type phenotype (Suzuki 1970). The times during which Pn.p cells are generated, undergo determination, and express a particular cell lineage are shown. The lin-10 TSP is not significantly different from the time of Pn.p cell determination; the apparent small difference is within the resolution of these experiments. 


\section{Identification of transposon insertions in lin-10}

The two mutator-derived lin-10 alleles, $n 1299$ and n1387, are candidates for containing Tc1 insertions in lin-10. Because the mutator strain contains several hundred Tcl copies (Emmons et al. 1983; Collins et al. 1987), we crossed the n1299 mutation into a genetic background with a low Tcl copy number to detect a new transposon insertion within the lin-10 locus by Southern blot analysis. lin-10(n1299) animals were crossed repeatedly with animals derived from the Bristol strain N2, which has only $30 \mathrm{Tcl}$ copies (Emmons et al. 1983). In each cross, the mutator-derived lin-10 mutation was retained, whereas other regions of the genome were gradually replaced with Bristol sequences (see Experimental procedures|. After eight crosses, a strain was obtained containing mutator-derived sequences in the region of lin-10(n1299) but Bristol sequences almost everywhere else.

Southern blot experiments indicated that this congenic strain contains three new $\mathrm{Tcl}$ copies that are linked to 1 in-10, and appear as novel 5.3-, 3.3- and 2.8-kb EcoRI bands (data not shown). Each Tcl insertion was genetically mapped using multifactor crosses to determine whether any were candidates for causing the lin-10 mutation in this strain (Table 1; and Fig. 3). In general, recombinants were obtained from heterozygotes carrying lin-10(n1299) in trans to visible flanking markers. Animals that were homozygous for the recombinant chromosome were scored for the presence of lin-10(n1299) and the three Tcl insertions (scored as novel Tc1 bands on Southern blots). A Tcl insertion into lin-10 should always be present in recombinants containing the lin-10(n1299) mutation and always absent in recombinants lacking it. The Tcl insertions associated with the 3.3- and 2.8-kb EcoRI bands do not represent insertions within lin-10, because both are present in some recombinants that are wild type for 1 in-10; we named these insertions $n P 46$ and $n P 47$, as they define polymorphisms between the mutator-derived and wildtype strains. However, the Tcl insertion associated with the 5.3-kb EcoRI band segregated with lin-10(n1299) in all recombinant animals, indicating that this $\mathrm{Tcl}$ copy maps within 0.03 map units of lin-10. Because the $C$. elegans genome size is $\sim 10^{8}$ bp (Sulston and Brenner 1974; J. Sulston, pers. comm.) and the genetic map has $\sim 300$ map units (Herman 1988), our genetic data suggest that this transposon is located within $\sim 10 \mathrm{~kb}$ of the lin-10(n1299) mutation.

We cloned the 5.3-kb EcoRI fragment containing the third Tcl insertion and used sequences flanking the Tc1 insertion to isolate the corresponding 3.7-kb EcoRI fragment from wild-type DNA, yielding plasmid p3.7 (see Experimental procedures). Using single copy sequences from $\mathrm{p} 3.7$ as a probe, we showed in Southern blot experiments that the 3.7-kb EcoRI fragment in wild-type DNA corresponds to a 6.9-kb EcoRI band in DNA from strains carrying the second lin-10 mutator allele, n1387 (data not shown). Surprisingly, the results of Southern blots using different enzymes indicate that this change in size is caused by the insertion of two $1.6-\mathrm{kb}$ elements, each of which has several restriction sites in common with $\mathrm{Tcl}$, suggesting that there are two Tc1 insertions associated with lin-10(n1387) (data not shown). One of these insertions is located within $50 \mathrm{bp}$ of the Tcl insertion in the 1 in-10(n1299) strain, whereas the other is $1 \mathrm{~kb}$ away (data not shown). The parental mutator strain does not contain a Tcl insertion in this EcoRI fragment (data not shown). We assume that one (or both) of these Tcl insertions is responsible for the lin-10(n1387) phenotype. Thus, the 1 in-10 mutations $n 1299$ and $n 1387$ both appear to be Tcl insertions.

To confirm further that the $\mathrm{Tcl}$ insertion associated with the 5.3-kb EcoRI fragment is the cause of the

Table 1. Mapping Tc1 insertions lin-10(n1299)

\begin{tabular}{|c|c|c|c|c|c|}
\hline $\begin{array}{l}\text { Genotype of } \\
\text { heterozygote }\end{array}$ & $\begin{array}{l}\text { Phenotype of } \\
\text { recombinant }\end{array}$ & $\begin{array}{l}\text { Genotype of } \\
\text { homozygous } \\
\text { recombinant }\end{array}$ & $n 1299::$ Tc1 & $n P 46$ & $n P 47$ \\
\hline$d p y-5+u n c-29$ & \multirow{2}{*}{ Dpy } & \multirow{2}{*}{$\begin{array}{l}d p y-5 \text { lin-10 } \\
d p y-5\end{array}$} & $12 / 12$ & $3 / 4$ & $7 / 8$ \\
\hline$+\operatorname{lin}-10+$ & & & $0 / 11$ & $0 / 8$ & $5 / 11$ \\
\hline & Unc-29 & unc-29lin-10 & $2 / 2$ & N.D. & $2 / 2$ \\
\hline unc $-13+$ sup -27 & \multirow{3}{*}{ Unc-13 } & unc-29 & $0 / 9$ & $2 / 4$ & $0 / 7$ \\
\hline & & unc-13 lin-10 & $1 / 1$ & N.A. & N.A. \\
\hline $\begin{array}{c}+ \text { lin }-10+ \\
\text { dpy-14 + unc-29 }\end{array}$ & & unc-13 & $0 / 5$ & N.A. & N.A. \\
\hline$\frac{1+\operatorname{lin}-10+}{x}$ & Unc-29 & $\begin{array}{l}\text { unc-29 lin-10 } \\
\text { unc-29 }\end{array}$ & $\begin{array}{l}8 / 8 \\
0 / 43\end{array}$ & $\begin{array}{l}\text { N.A. } \\
\text { N.A. }\end{array}$ & $\begin{array}{l}\text { N.A. } \\
\text { N.A. }\end{array}$ \\
\hline
\end{tabular}

In general, $\mathrm{X}$ non- $\mathrm{Y}$ and $\mathrm{Y}$ non-X recombinants were isolated from the $\mathrm{F} 1$ progeny of $\mathrm{X}+\mathrm{Y} /+\operatorname{lin}-10+$ heterozygotes. Homozygous recombinants segregated in the F2 generation were then scored for lin-10(n1299). DNA from these homozygous recombinants was analyzed by Southern blot analysis to test for the presence of $n 1299:: T c 1, n P 46$, and $n P 47$. A Tcl copy inserted within lin-10 should always segregate with $n 1299$. Fractions of recombinant animals that contain a particular Tc1 copy are shown. $n 1299::$ Tc1, nP46, and $n P 47$ were scored as novel 5.3-, 3.3-, and 2.8-kb Tcl-containing EcoRI fragments, respectively. Some blots used single-copy lin-10 sequences as a probe (a gel purified 1.8-kb PvuII fragment derived from p3.7); thus, the detection of $n P 46$ and nP47 polymorphisms is not applicable (N.A.). (N.D.) Not done. One $d p y-5$ lin- 10 recombinant did not segregate the $n P 47$ polymorphism and may be the result of a double recombination event or excision of the $n P 47 \mathrm{Tc} 1$ element. 


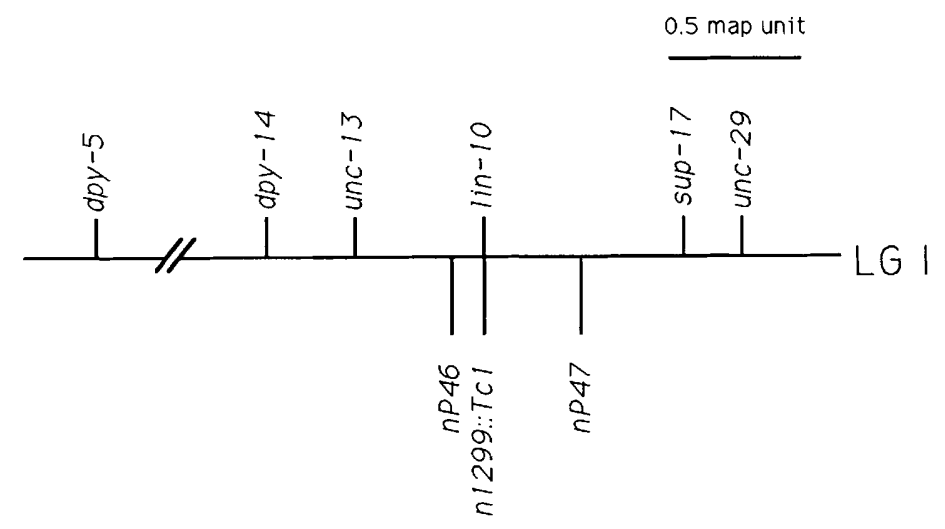

Figure 3. Genetic map of $\mathrm{Tc1}$ insertions near lin-10(n1299). The genetic positions of two Tcl polymorphisms ( $n P 46$ and nP47) as well as the Tcl insertion into lin-10 (n1299:: Tc1) are shown below the line, using data from Table 1. Genetic markers are indicated above the line. lin-10(n1299) mutation, we isolated an intragenic revertant. In mutator strains, transposon-insertion alleles can revert spontaneously as a result of the excision of the integrated transposon (Eide and Anderson 1988; Kiff et al. 1988; Rosenquist and Kimble 1988; Way and Chalfie 1988). Two non-vulvaless revertants were isolated from the original lin-10(n1299) strain, which is still active for transposition (see Experimental procedures). One revertant carried a mutation, $n 1326$, that is tightly linked to lin-10 (within 2.2 map units) and has a dominant suppressing phenotype, suggesting that it is an intragenic revertant (see Experimental procedures). As shown by Southern blot experiments, n1299 n1326 animals no longer contain the Tcl transposon insertion associated with the 5.3-kb EcoRI fragment (Fig. 4A). Thus, reversion of $\operatorname{lin}-10(\mathrm{n} 1299)$ is associated with the excision of the Tcl insertion, indicating that this insertion is the cause of the lin-10(n1299) mutation. We refer to this

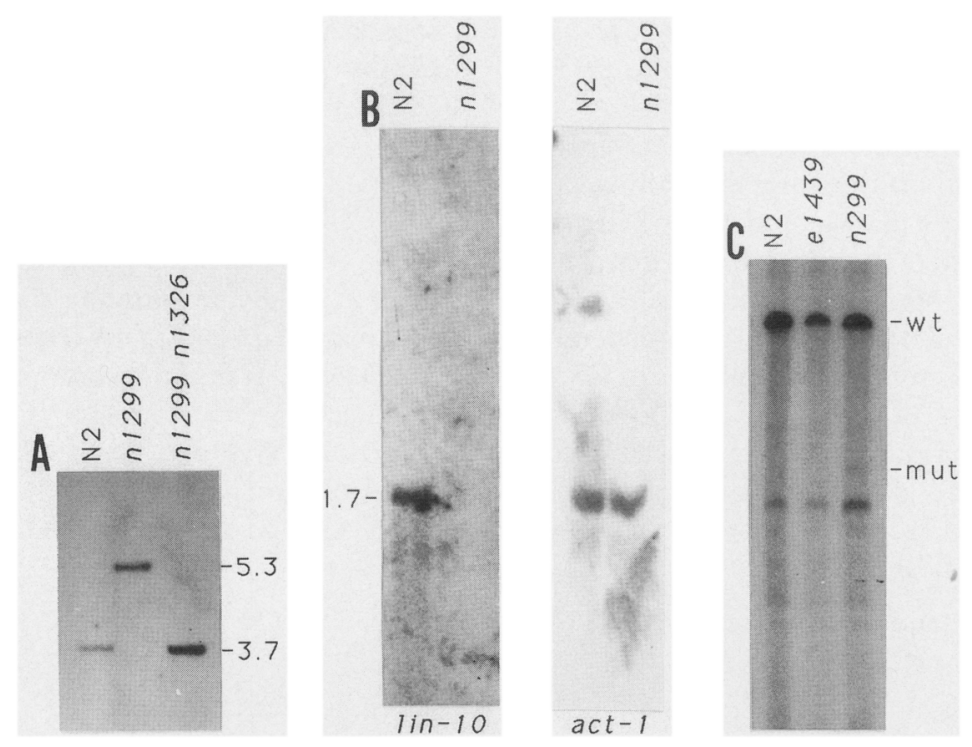

Figure 4. (A) Excision of $n 1299::$ Tc1 from an intragenic lin-10(n1299) revertant. A Southern blot is shown analyzing DNA from the wild-type, lin-10(n1299) animals and intragenic revertant lin-10(n1299 n1326) animals. DNA was isolated, digested with EcoRI, blotted onto a Nylon membrane and hybridized with a 1.8-kb Pvull fragment (see Experimental procedures), representing single-copy DNA flanking the $n 1299:: T c 1$ insertion. $(B)$ Identification of lin-10 RNA by Northern blot analysis. Poly(A) ${ }^{+}$RNA from wild-type and lin-10(n1299) animals was analyzed by Northern blotting. A 1.7-kb putative lin-10 RNA was detected by hybridization with p3.7. The filter was stripped and then hybridized with an actin probe, act-1, showing that RNA is present in both lanes. Northern blot experiments using RNA isolated from animals carrying the other mutator-induced lin-10 allele, $n 1387::$ Tc1, gave similar results in which the 1.7-kb RNA band was reduced or eliminated in intensity (S. Kim, unpubl.). The size of the lin-10 mRNA was estimated using actin mRNA and rRNAs as size markers. $(C)$ Detection of a point mutation in $n 299$ RNA by RNase A protection. An antisense RNA probe containing 420 bp of lin- 10 sequence was synthesized from a lin-10 cDNA clone, c10-7 (see Experimental procedures), and hybridized to $20 \mu \mathrm{g}$ of total RNA from wild-type and n299 animals at mixed stages. Unhybridized RNA, as well as RNA near a single-base mismatch, was digested with RNase A. Protected fragments were resolved on a $6 \%$ denaturing polyacrylamide gel and visualized by autoradiography. (wt) The main band corresponding to protection of all $420 \mathrm{bp}$ of the lin-10 sequence; (mut), an extra band in $n 299$ RNA, presumably indicating partial cleavage that results from an RNA mismatch. The band below that designated mut is probably a result of cleavage at a region of high AT content by RNase A (S. Kim, unpubl.). 
Tc1 insertion as $n 1299:: T c 1$. The second revertant carried an extragenic suppressor mutation, n1329, and will not be discussed further in this paper.

\section{Identification of a lin-10 transcript}

A 1.7-kb poly $(\mathrm{A})^{+}$RNA was detected in Northern blot experiments using sequences flanking the $n 1299::$ Tc1 insertion site as a probe (Fig. 4B). As shown below, this transcript is likely to be the lin-10 mRNA. We screened several $C$. elegans cDNA libraries, using p3.7 as a probe, and obtained $23 \mathrm{cDNA}$ clones representing this RNA (see Experimental procedures). The 1.7-kb RNA is a rare message; it is 50 times less abundant than actin RNA, and the cDNA clones were isolated at a low frequency (15 lin-10 cDNA clones per 250,000 total cDNA clones). However, the abundance of this transcript may be higher in particular cells, as our experiments reflect the level of expression in the entire animal. The longest cDNA clones hybridize to three regions of genomic DNA spaced over an interval of $14 \mathrm{~kb}$ (Fig. 5 and data not shown). These three regions may each correspond to a single exon because only three fragments were protected in RNase protection experiments, in which genomic DNAs were hybridized to a single-stranded RNA probe derived from a cDNA clone (Fig. 5 and data not shown). The gene may extend beyond this $14-\mathrm{kb}$ interval, as the precise $5^{\prime}$ end has not been determined.

We have found no evidence for a second gene in the vicinity of the $n 1299:: T c 1$ insertion site, using sequences extending $4 \mathrm{~kb}$ on either side of the $\mathrm{Tcl}$ insertion site as probes in Northern blotting experiments or in screening cDNA libraries (data not shown). Furthermore, we have no evidence that the $14-\mathrm{kb}$ gene undergoes differential RNA splicing, because all of our cDNA clones have colinear restriction maps and only one RNA band is observed in Northern blots.

The 14-kb gene is likely to be $l i n-10$, as it contains three lin-10 mutations. In animals carrying either of the Tcl insertion alleles ( 1299 or $n 1387$ ), the level of expression of the $14-\mathrm{kb}$ gene is greatly reduced or eliminated (Fig. 4B and data not shown). The Tcl insertion sites of both $n 1299$ and $n 1387$ occur within an intron (Fig. 5), indicating that these insertions probably interfere with either the proper transcription of the $14-\mathrm{kb}$ gene or the splicing of its primary RNA. Furthermore, we have found that a strain carrying the ethylmethane sulfonate (EMS)-induced allele lin-10(n299) has a point mutation in the $14-\mathrm{kb}$ gene. To detect lin-10 point mutations, we used an RNase protection assay (Myers et al. 1985; Winter et al. 1985) in which an antisense RNA probe was synthesized from a lin-10 cDNA clone and hybridized to RNA from either the wild-type or mutant lin-10 strains. Wild-type RNA duplexes are protected from digestion with RNase A, whereas RNA duplexes containing a single mismatch may be cleaved. We searched for point mutations in five different EMS-induced lin-10 alleles (e1438, e1439, n299, n1386 and n1390|. In four cases, mutations were not detected, perhaps because the probe did not cover the mutation (the probe covers only about one-third of the RNA) or because RNase A was not able to cleave the mismatched base pair (RNase $\mathrm{A}$ is able to cleave about $25 \%$ of all combinations of mismatched base pairs; Myers et al. 1985). However, a novel band was observed in the case of $n 299$, indicating that this RNA contains a point mutation (Fig. $4 \mathrm{C}$ ). The finding of the point mutation is important, because this mutation probably affects only the protein expressed from the 1.7-kb mRNA, unlike transposon insertions, which can affect the expression of nearby genes in a complicated fashion (Lipshitz at al. 1987).

\section{Rescue of the lin-10 mutant phenotype by germ line transformation}

Further evidence showing that the $14-\mathrm{kb}$ gene is $\operatorname{lin}-10$ was obtained from DNA transformation experiments. In C. elegans, transgenic animals can be obtained by microinjecting DNA into oocytes (Stinchcomb et al. 1985; Fire 1986; Way and Chalfie 1988). Genes introduced in this fashion can be correctly regulated, and unselected DNA can be cotransformed with selected DNA (Fire 1986).

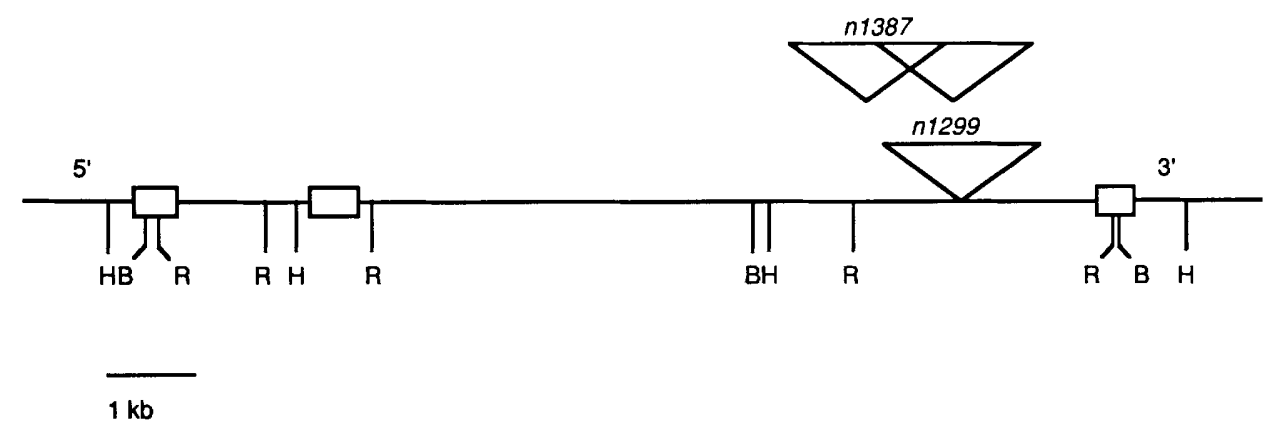

Figure 5. Physical map of the lin-10 locus. The boxes represent the three exons; their positions were derived by the regions of genomic DNA that hybridized to lin-10 cDNA probes. The exact position of the second exon is not known, although it must be located between the EcoRI and HindIII sites shown. The first and third exons each contain EcoRI and BamHI sites, allowing their exact positions to be determined. The single Tc1 insertion site in $n 1299$ strains and the two Tc1 insertion sites in $n 1387$ strains are shown. The positions of these Tcl insertions were determined by Southern blot analysis. (R) EcoRI; $(\mathrm{B})$ BamHI; (H) HindIII. 
In preliminary experiments, cosmids spanning the $14-\mathrm{kb}$ gene were introduced into $\operatorname{lin}-10(\mathrm{n} 1390)$ animals, resulting in transformants that were partially rescued for the vulvaless phenotype. lin-10(n1390) is incompletely penetrant: Out of 3765 animals, $95.6 \%$ were vulvaless, $4.1 \%$ exhibited a protruding vulva (indicative of a weak lin-10 phenotypel, and $0.3 \%$ exhibited a wild-type phenotype. In transgenic strains, over half of the animals exhibited complete or partial rescue: $45 \%$ were vulvaless, $50 \%$ exhibited a protruding vulva and $5 \%$ exhibited a wild-type phenotype (300-500 animals were counted for each of 25 transgenic strains). These experiments have never generated a strain in which all of the transformants are completely rescued for the lin-10 mutant phenotype, even though we have obtained many transformants using four different cosmid clones containing the 14-kb gene (B234, AAE8, K7E6, and ZK265). It is not clear why these animals are incompletely rescued. (Complete rescue has been observed for other C. elegans genes, such as mec-3; Way and Chalfie 1988.) Perhaps all four cosmids lack sequences required for proper lin-10 expression. For example, lin-10 may utilize distant regulatory sequences, as do the $\beta$-globin and Ultrabithorax genes (Grosveld et al. 1987; Peifer et al. 1987). Alternatively, lin-10 may be poorly expressed from transgenes, perhaps because they are usually present as extrachromosomal arrays (Stinchcomb et al. 1985; Fire 1986; Way and Chalfie 1988).

The region encoding lin-10 activity within one of the cosmids, B234, was defined by determining which restriction enzymes reduce transforming activity and thus presumably cut within lin-10. In these experiments, B234 was cut with a particular restriction enzyme and then coinjected with a marker gene, unc-31(+) (contained on the cosmid C14G10; R. Hoskins, pers. comm.l, into lin-10; unc-31 mutant animals. Transfor- mants were identified because they were non-Unc and segregated non-Unc progeny; the Lin phenotype of these transformants was subsequently determined. In control experiments using the intact lin-10 cosmid B234, we found that about half of the unc-31 + () transformants were also rescued for the lin-10 vulvaless phenotype (Fig. 6). Cotransformation occurred at a similar frequency when B234 was cut with NruI, MluI, SalI, Tth111I, or SphI (Fig. 6), suggesting that these enzymes do not cut within lin-10. Because lin-10 is presumably contained on the same fragment as the $n 1299::$ Tc1 insertion site, these data define a $24-\mathrm{kb}$ region containing lin-10 transforming activity, bounded by an SphI site on one side and an NruI site on the other. This region contains the entire $14-\mathrm{kb}$ gene.

When cosmid B234 was cut with SacII, NheI or ApaLI, rescuing activity was lost. These enzymes cut within the 14-kb gene; the NheI site is located $4 \mathrm{~kb}$ from its $5^{\prime}$ end and the SacII site is located $1 \mathrm{~kb}$ from its $3^{\prime}$ end. Thus, when the 14-kb gene is intact, lin-10-transforming activity is present, but removal of sequences from either end severely reduces rescuing activity. These results indicate that the $14-\mathrm{kb}$ gene is $\operatorname{lin}-10$.

\section{lin-10 sequence}

We determined the DNA sequence of four independent lin-10 cDNA clones: c10-6, c10-7, c10-12, and cl0-13. The inserts in three of these clones (c10-6, c10-12, and c10-13) may represent most of the lin-10 mRNA, as they are approximately the size expected for full-length clones; they each contain $\sim 1507 \mathrm{bp}$ and thus probably correspond to almost all of a 1700-bp mRNA (as seen in our Northern blot experiments) when a poly(A) tail is included. The lin-10 sequence has one long open reading frame, encoding 421 amino acids (Fig. 7). This open
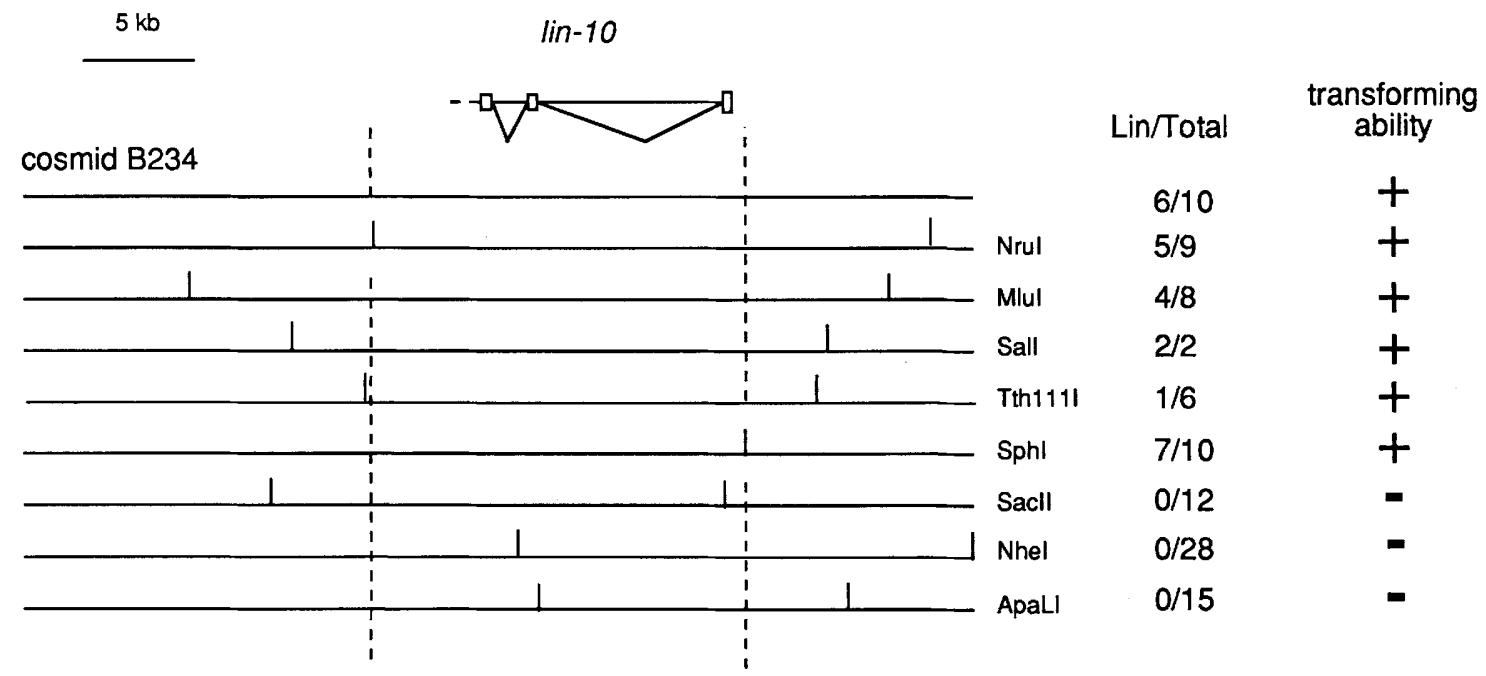

Figure 6. lin-10 transformation. Cosmid B234 was digested with various restriction enzymes and then coinjected with unc-31( + ) DNA into lin-10; unc-31 mutants. The number of Lin transformants obtained from the total number of Unc transformants is expressed as a fraction for each restriction enzyme tested. |+ and - | The transforming ability of B234 after restriction with a particular enzyme. The vertical dashed lines represent the minimal region of DNA sufficient to rescue the lin-10 mutant phenotype. The position of the 14-kb gene is shown (top). 
reading frame extends to the $5^{\prime}$ end of the sequence, indicating that the beginning of the 1 in- 10 protein sequence may not be included in these clones. The predicted protein sequence does not have any striking structural motifs, such as a transmembrane-spanning segment. When this sequence was compared to other sequences in several data bases (see Experimental procedures), no significant similarity was found, suggesting that lin-10 may define a new class of developmental control gene. lin-10 is expressed in cells other than Pn.p cells

We have investigated the pattern of 1 in-10 expression by determining the level of lin-10 RNA in mutant animals that lack particular cells. First, we asked whether the anchor cell signal, which induces vulval cell lineages, is needed to activate 1 in-10 expression. For example, the expression of lin-10 may induce P5.p, P6.p, and P7.p to undergo vulval cell lineages. To test this possibility, we examined the level of lin-10 expression in lin-12(n302)

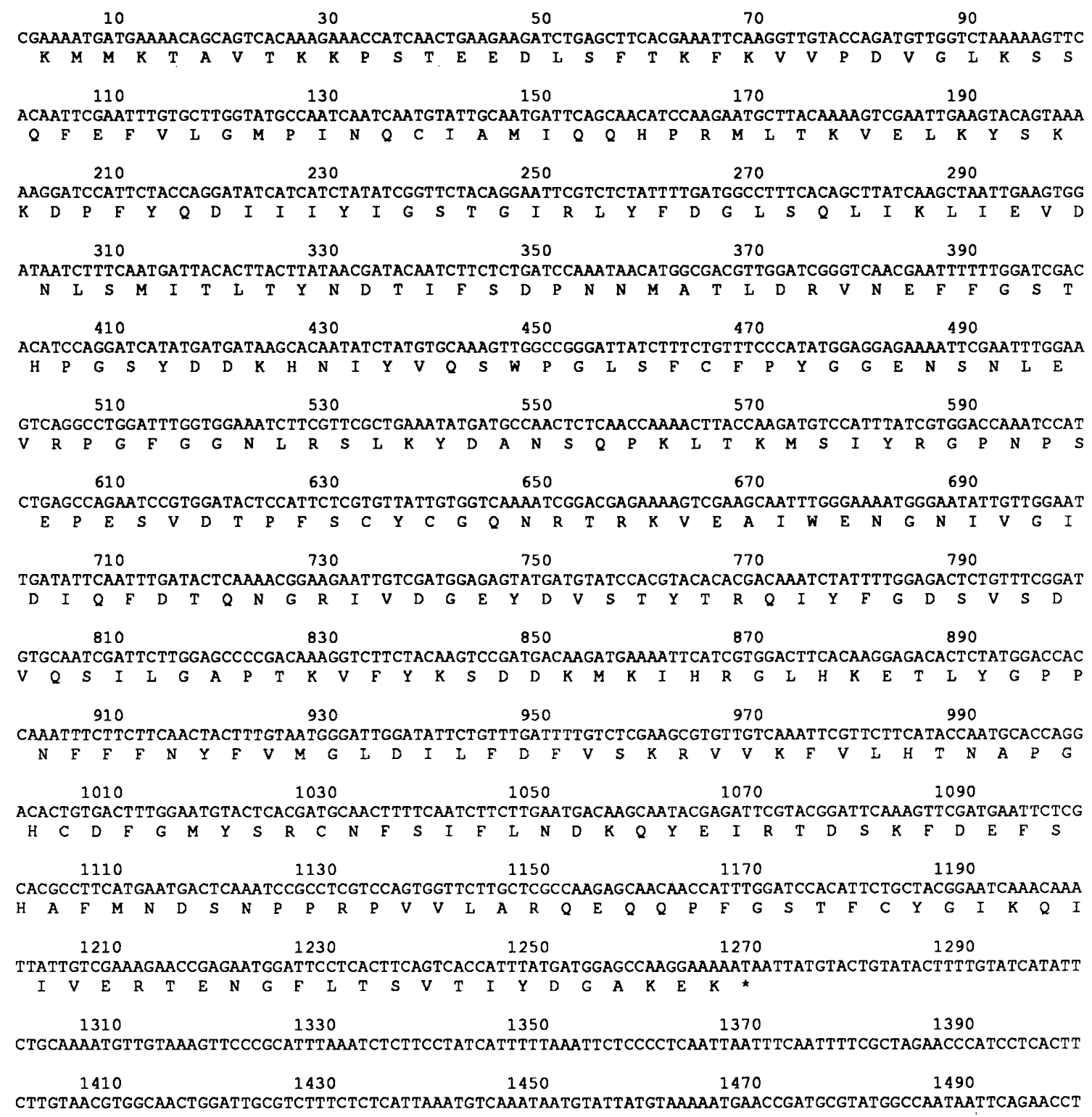

ATGTGGA

Figure 7. The lin-10 sequence. The lin-10 nucleotide and predicted amino acid sequences are shown, based on the sequences of four independently isolated lin-10 cDNA clones (c10-6, c10-7, c10-12, and c10-13). The open reading frame terminates at the stop codon beginning at base 1272. c10-13 has a 9-bp deletion beginning at position 255; otherwise, these clones share identical sequences over their regions of overlap. c10-6, c10-12, and c10-13 each cover essentially all of the 1507 bp presented. c10-7 contains the terminal 822 bp at the $3^{\prime}$ end. 
mutant animals, which lack the anchor cell /see Experimental procedures). [The lin-12(n302) mutation causes a transformation of the anchor cell into a ventral uterine cell; because the anchor cell is missing, all Pn.p cells express the $3^{\circ}$ cell lineage, resulting in a vulvaless phenotype (Greenwald et al. 1983)]. The level of lin-10 expression was determined by an RNase protection assay, using an actin probe, act-1, as a control to show that approximately equal levels of RNA were loaded in each lane. As seen in Figure 8a, the level of lin-10 RNA is unchanged in lin-12(n302) animals relative to wild-type animals, indicating that lin-10 is not expressed exclusively in response to the reception of the anchor cell signal.

Second, because lin-10 appears to be required only for the expression of vulval cell lineages by the vulval precursor cells, lin-10 might be expressed specifically in these cells. For example, genes involved in transducing the anchor cell signal could be expressed in all six vulval precursor cells but in no other cells, as only these six cells have the potential to undergo vulval cell lineages. We measured the level of $l i n-10$ expression in mutants that lack these cells (Ferguson et al. 1987). In lin-26 animals, P3.p-P8.p. are transformed into neurons or neuroblasts. In $n 300$ animals, these cells are transformed into ventral hypodermal cells that do not have the potential to express vulval cell lineages. Using the RNase protection assay described above, the levels of lin-10 expression in lin-26 and $n 300$ mutants were found to be similar to that in wild-type animals (Fig. 8a). This result indicates that $7 \mathrm{in}-10$ is expressed in cells other than P3-P8.p.

Third, lin-10 mRNA could be expressed in Pn cells, which give rise to Pn.p cells, resulting in the production of a gene product that functions in Pn.p cells. Accordingly, we measured the level of lin-10 expression in mutants that lack Pn cells. Normally, Pn cell nuclei migrate from the lateral to the ventral hypodermis during the first larval stage (Sulston and Horvitz 1977). In unc- 83 and unc- 84 mutants, this migration is defective and almost all Pn cells degenerate and die (Sulston and Horvitz; 1981). In these mutants, lin-10 expression is the same as in wild-type animals, indicating that cells other than Pn cells and their progeny must express lin-10 (Fig. 8A).

Finally, we have determined the level of lin-10 expression during different developmental stages. RNA was prepared from staged hermaphrodites, and the level of lin-10 expression at each developmental stage was determined by RNase protection. As shown in Figure 8B, lin-10 is expressed throughout development, and embryonic expression is severalfold higher than either larval or adult expression. Pn.p cells are born in the first larval stage and become committed to express a particular cell
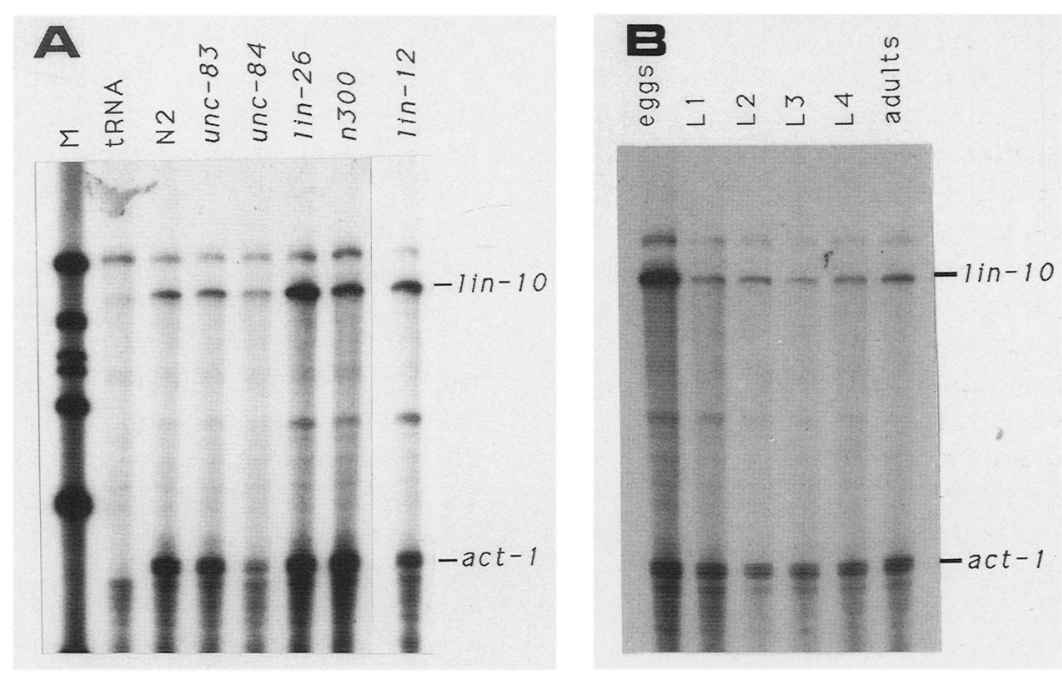

Figure 8. (A)lin-10 expression in mutants lacking Pn.p cells. RNA $(20 \mu \mathrm{g})$ from each of the designated mutants was analyzed by RNase protection using the probe and procedure described in Fig. 4C. An actin probe act-1, was included as a normalization control to show that equal amounts of RNA had been loaded into each lane. The act-1 probe was synthesized with one-fiftieth the specific radioactivity of the lin-10 probe to compensate for the greater abundance of act-1 over lin-10 mRNA. The band at 520 bp corresponds to undigested input probe. (M) size markers $(517,396,360,344,298,220$, and $179 \mathrm{bp}$ ); (tRNA) yeast tRNA negative control; (N2) wild-type C. elegans RNA. RNAs derived from mutants were isolated from strains carrying one of the following mutations: unc-83(e1409), unc-84(e1410), lin-26(n156), n300 or lin-12(n302). By itself, the experiment involving lin-12(n302) animals does not show that 1 in-10 is expressed independently of the anchor cell signal, as the lin-12(n302) mutation may result in the partial induction of vulval cell lineages (Greenwald et al. 1983). However, subsequent experiments shown here and in $B$ confirm our assertion, indicating that lin-10 is expressed in cells other than Pn.p cells and therefore must be expressed independently of the anchor cell signal. (B) Developmental timing of lin-10 expression. Staged RNA was prepared from synchronous wild-type hermaphrodites at different developmental stages (see Experimental procedures). The steady-state level of lin-10 and act-1 expression at each developmental stage was determined using the RNase protection assay described in Fig. 4C and the probes described in $(A)$. (L1-L4), First larval stage to fourth larval stage. 
fate in the third larval stage. Thus, lin-10 is expressed both before Pn.p cells are born and after they undergo determination.

\section{Discussion}

We have presented a genetic and molecular analysis of lin-10, a gene involved in the specification of Pn.p cell fates. We used transposon-insertion mutagenesis to clone this gene and demonstrated that the cloned DNA can rescue the lin-10 vulvaless defect in germ line transformation experiments. The predicted protein sequence of this gene is not similar to previously characterized gene sequences, suggesting that 1 in-10 may have a novel role in the specification of cell fates.

Although lin-10 is required specifically for Pn.p cell determination, this gene is expressed more generally than in these cells alone and at developmental stages when these cells are not present. How can we interpret the presence of lin-10 transcripts in cells that are not developmentally affected by the elimination of $\operatorname{lin}-10$ gene activity?

First, it is possible that the functional lin-10 gene product may not be encoded by the 1.7-kb mRNA, but rather by another mRNA produced by differential RNA splicing from the 14-kb gene. Alternative RNA splicing has been shown to regulate the production of functional P-element transposase in Drosophila; functional transposase is not produced by the predominant P-element splicing pattern, but rather from a rare RNA splicing variant present only in the germ line (Laski et al. 1986). By analogy, it is possible that a functional lin-10 gene product is produced only in Pn.p cells because these are the only cells that express a rare RNA splicing variant. Our experiments have not detected any such alternatively spliced RNA, and we estimate that the Northern blot experiment presented in Figure $4 \mathrm{~B}$ and the RNase protection assay shown in Figure $8 \mathrm{~B}$ would detect another RNA form if it were present at $5 \%$ of the level of the $1.7-\mathrm{kb}$ RNA. The possibility that the functional lin-10 gene product is encoded by a rare RNA splicing variant leaves open questions regarding the function of the polypeptide encoded by the $1.7-\mathrm{kb}$ mRNA.

A second possibility is that lin-10 may function more generally than in Pn.p cells but that its function in these other cells is dispensable. Numerous C. elegans genes are known in which the elimination of gene function results in no obvious phenotypic abnormalities (Greenwald and Horvitz 1980; Park and Horvitz 1986). One possibility is that these genes have redundant activities, such that the elimination of the activity of one gene is compensated by a redundant activity encoded by another gene or genes. In such cases, independent mutations in two genes may be required to remove both activities and thus produce a mutant phenotype. For example, the multivulva phenotype can result from a combination of mutations in each of two redundant pathways; mutations affecting only one of these pathways result in a wild-type phenotype /Horvitz and Sulston 1980; Ferguson et al. 1987; Ferguson and Hor- vitz 1989|. By analogy, lin-10 mutations may not affect the development of cells other than Pn.p cells because lin-10 (or a pathway involving lin-10) may be functionally redundant with another gene (or gene pathway) in these other cells.

Third, lin-10 may indeed function only in Pn.p cells, even though it is expressed elsewhere. Any type of posttranscriptional regulation could restrict lin-10 action in this way. For example, post-translational modification could limit the activity of the lin-10 protein to Pn.p cells. Alternatively, the activity of the lin-10 protein might be widespread but have biological consequences only in conjunction with the products of other genes. In this case, lin-10 may act only within Pn.p cells because these cells may be the only ones in which all of the gene products required for 1 in-10 to function are present. A similar situation may exist in Drosophila sex determination, in which the tra-2 gene may function somatically only in females even though it is expressed in both sexes, because its function may be dependent on the presence of the female-specific product of the tra gene (Amrein et al. 1988; Goralski et al. 1989). Finally, lin-10 may act only in Pn.p cells because only these cells receive an inductive signal from the anchor cell. Genes that function in signal transduction could well act in this way. The sevenless gene in Drosophila illustrates this point, because loss-of-function mutations in this gene specifically affect the development of the R7 cells in the eye (Harris et al. 1976), and yet this gene is expressed in many cells other than R7 cells (Banerjee et al. 1987; Tomlinson et al. 1987). sevenless encodes a tyrosine kinase and may function in a signaling pathway that specifies the R7 cell fate (Hafen et al. 1987). The mutant phenotype for genes such as sevenless may reflect which cells receive an inductive signal, whereas their pattern of gene expression may indicate which cells have the potential to respond to that signal.

The issue of whether lin-10 functions solely in Pn.p cells raises an important question regarding the logic of the network that regulates cell fates: are there genes that specify one, and only one, cell type? Most vulval determination genes have pleiotropic mutant phenotypes; for example, mutations in lin-12, lin-15, and lin-31 each result in abnormal cell lineages in the male tail, as well as the hermaphrodite vulva (Greenwald et al. 1983; Ferguson and Horvitz 1985; P. Sternberg, M. Herman; both pers. comm.). None of these genes acts to specify vulval cell fates exclusively. Rather, the genes are likely to control multiple lineages by acting in combination with other genes, such that their interactions specify a vulval cell fate in some contexts and a male tail cell fate in others. Of the 17 vulval determination genes that have been identified, mutations in 14 affect multiple cell lineages, whereas loss-of-function mutations in only 3 (lin-2, lin-7, and lin-10) appear to affect Pn.p cell fates solely (Ferguson and Horvitz 1985). Thus, only these three genes could potentially specify one, and only one, cell type. However, our molecular studies raise the possibility that at least $\operatorname{lin}-10$, and perhaps $\operatorname{lin}-2$ and $\operatorname{lin}-7$ as well, may function in diverse cell types. 
Vulval development has been studied extensively at the cell lineage and genetic levels. These studies have established a framework for a molecular dissection of the process of cellular determination, because they have identified many steps in the pathway for the specification of Pn.p cell fates. As exemplified with lin-10, these genes can be directly cloned by transposon tagging. We hope to extend our molecular analysis of the vulval determination pathway using our mutator-induced alleles to clone lin-2, lin-15, and lin-31. We expect many of the vulval determination genes to be structurally and functionally related to genes characterized previously in other systems. For example, lin-12 is similar to the mammalian LDL receptor gene, the Drosophila Notch gene and the yeast SWI6 gene (Greenwald 1985; Breeden and Nasmyth 1987; Yochem et al. 1988). The products of other genes, such as lin-10, may have structures that are novel. An analysis of these novel genes, in the context of a pathway in which they interact with genes with familiar sequence motifs, should be instrumental in extending our knowledge of how cell fates are specified during development.

\section{Experimental procedures}

\section{General methods and strains}

C. elegans strains were maintained as described by Brenner (1974) at $20^{\circ} \mathrm{C}$, unless otherwise noted. C. elegans strain variety Bristol N2 (Brenner 1974) was the wild-type parent of all strains used in this work, and TR679 is a hybrid Bristol/Bergerac strain containing the mut-2(r453) mutation (Collins et al. 1987). The genetic markers used were as follows (unless otherwise noted, all mutations are listed by Hodgkin et al. 1988): LGI: dpy-5(e61), dpy-14(e188), unc-13(e1091), unc-15(e73), fer-1(hc1), sup-17(n316), unc-29(e1072, e403), lin-11(n389), unc-75(e950) and the previously identified lin-10 alleles (e1438, e1439, n299) (Horvitz and Sulston 1980; Ferguson and Horvitz 1985 ) as well as the lin-10 alleles identified in this work [ $n 1386$ (G. Garriga, pers. comm.), n1387, n1402, n1853 (S. Clark, pers. comm.), n1390 (M. Stern, pers. comm.), n1299, n1299n1326, n1507, n1508, n1509ts, n1511, n1530, n1541, n1636, n1637, n1638am (this work)]; LGII: lin-31(n301) as well as the new alleles defined in this work (n1282, n1289, n1290, n1291, n1292, n1293, n1294, n1300, n1301), lin-26(n156); LGIII: dpy-19(e1259), sup-5(e1464), lin-12(n302, n950) as well as the new allele defined in this work (n1295); LGIV: unc-31(e928); LGV: unc-83(e1409), him-5(e1490); LGX: lon-2(e678), lin-2(e1309) as well as the new alleles defined in this study (n1297, n1298), unc-84(e1410), lin-15 (n309) as well as the new alleles defined in this study $(n 1296, n 1331, n 1334) . n 300$ is a mutation associated with the reciprocal translocation $n T 1(I V ; V)$.

\section{Isolation of lin-10 alleles by non-complementation}

New lin-10 alleles were isolated in three ways by their failure to complement a pre-existing lin-10 allele, e1439. First, lin-10(e1439); him-5 males were mated with dpy-5 unc-29(e1072) hermaphrodites that had been mutagenized with EMS (Brenner 1974). Most cross progeny would be wild type, whereas a rare animal carrying a new lin-10 mutation would be vulvaless. One such animal was isolated after screening 5500 cross progeny. This animal segregated Dpy Lin Unc progeny, of genotype dpy-5 lin-10(n1511) unc-29(e1072). In a second screen, unc-13 lin-10(e1439)/lin-10(e1439) unc-29(e1072); him- 5 males were mated with EMS-mutagenized unc-15 fer-1 unc-75 hermaphrodites. From $16,000 \mathrm{~F}_{1}$ cross progeny, three vulvaless animals were isolated, defining three new lin-10 alleles: $n 1507, n 1508$ and $n 1509$ ts. These animals each segregated unc-15 lin-10 fer-1 unc-75 homozygous progeny. Third, EMS-mutagenized unc-29(e1072) males were mated with unc-13 lin-10(e1439); n1329 lon-2 hermaphrodites at $25^{\circ} \mathrm{C}$. (n1329 is a mutation that suppresses the vulvaless lin-10 phenotype and is linked to lon-2 on the X chromosome; S. Kim, unpubl.). The lon-2 mutation was included to prevent males from mating with hermaphrodites in the $F_{1}$ generation, since Lon males mate inefficiently at $25^{\circ} \mathrm{C}$ (Hodgkin et al. 1988). From 31,000 cross progeny hermaphrodites, four vulvaless animals were picked, and each segregated Unc-29 Lin progeny, thus defining four new lin-10 alleles $[n 1541, n 1636, n 1637$ and $n 1638 \mathrm{am}$ ). All eight of the new lin-10 alleles obtained in this non-complementation screen result in a recessive vulvaless phenotype and are linked to lin-10.

We tested four lin-10 alleles for amber suppression: $n 1508$, $n 1541, n 1637$, and n1638. lin-10 males were mated into dpy-19 sup-5(e1464) hermaphrodites, and individual cross-progeny $F_{1}$ heterozygotes were picked. [sup-5(e1464) encodes an amber suppressor tRNA and is tightly linked to $d p y-19$ on chromosome III (Wills et al. 1983)]. About 25 Lin animals segregated in the $F_{2}$ generation from these heterozygotes were individually placed on petri plates. For the experiments using n1508, n1541, and $n 1637$, these animals often segregated Dpy progeny that were also vulvaless, indicating that these lin-10 alleles are not suppressed by either one or two copies of sup-5(e1464). For the experiment using n1638, of 25 animals, none segregated $d p y-19$, suggesting that this allele is suppressed by one copy of sup-5(e1464). To confirm this result, we constructed a strain homozygous for both lin-10(n1638) and sup-5(e1464). lin-10(n1638) unc-29(e403)/ + heterozygous males were mated with dpy-19 sup-5(e1464) hermaphrodites (unc-29 is linked to lin-10). About half of the wild-type cross progeny segregated non-Lin Unc Dpy animals, which were presumably of genotype lin-10(n1638) unc-29(e403); dpy-19 sup-5(e1464). We picked one such animal and showed that it was homozygous for lin-10(n1638) by mating it with $\mathrm{N} 2$ males, picking 10 cross progeny hermaphrodites, and showing that each hermaphrodite segregated vulvaless animals.

As with the pre-existing alleles, all lin-10 alleles isolated in this study are incompletely penetrant to some extent, suggesting that lin-10 may not be absolutely required for the induction of vulval cell lineages. Specifically, the percentage of animals that cannot lay eggs is $99 \%$ for $n 1299$ ( $\mathrm{N}=550), 96 \%$ for $n 1386(\mathrm{~N}=420), 99 \%$ for $n 1387(\mathrm{~N}=350), 99.7 \%$ for $n 1390(\mathrm{~N}=3765), 99 \%$ for $n 1402$ ( $\mathrm{N}=780), 95 \%$ for $n 1507$ $(\mathrm{N}=430), 99.5 \%$ for $n 1508 \quad(\mathrm{~N}=2770), 70 \%$ for $n 1511$ $(\mathrm{N}=340), 95 \%$ for $n 1530(\mathrm{~N}=320), 97 \%$ for $n 1541(\mathrm{~N}=215)$, $95 \%$ for $n 1636(\mathrm{~N}=270), 95 \%$ for $n 1637(\mathrm{~N}=580), 98 \%$ for $n 1638(\mathrm{~N}=540), 99 \%$ for $n 1853(\mathrm{~N}=630), 30 \%$ for $n 1509$ at $15^{\circ} \mathrm{C}(\mathrm{N}=880)$, and $75 \%$ for $n 1509$ at $25^{\circ} \mathrm{C}(\mathrm{N}=950)$.

Other than a vulval defect, no other defect in hermaphrodite development, behavior, or morphology has been observed at the dissecting microscope level in lin-10 mutants. Furthermore, anatomical checks of specific cells have not revealed defects other than in cells involved in vulval development in lin-10(e1439) or lin10(n1390) hermaphrodites; cells that appear normal in these mutants include the NSM neurons (R. Ellis, pers. comm.), the HSN neurons (G. Garriga, pers. comm.), the sex muscles ( $M$. Stern, pers. comm.) and the VC neurons (C. Li, pers. comm.). 


\section{Temperature-shift experiments}

The TSP for lin-10(n1509ts) was determined by shifting animals from the permissive $\left(15^{\circ} \mathrm{C}\right)$ to the nonpermissive $\left(25^{\circ} \mathrm{C}\right)$ temperature, and vice versa, at various times during development. Temperature shifts for the embryonic, first larval, and fourth larval stages were performed by picking individuals developing at one temperature and shifting them to a new temperature. For more precise time points during the second and third larval stages, a synchronous population was obtained by placing phenotypically wild-type $n 1509$ adults (n1509 is incompletely penetrant at all temperatures) on a petri plate and allowing them to lay eggs for a short time. For the upshift experiments, 20 adult hermaphrodites were placed on a plate and allowed to lay eggs for $1.5 \mathrm{hr}$. For the downshift experiments, 10 adults were allowed to lay eggs for $1 \mathrm{hr}$. The temperature of the synchronous population was shifted at precise times during development, as determined by observing the morphology and size of the gonad of several worms with Nomarski optics (Kimble and Hirsh 1979). About 200-400 animals were counted for each time point. Animals that could not lay eggs were scored as mutant.

Isolation and characterization of putative

transposon-insertion alleles of vulval determination genes

Mutants with abnormal vulval lineages can be isolated by screening for multivulva and vulvaless animals, using a dissecting microscope (Horvitz and Sulston 1980; Greenwald et al. 1983; Ferguson and Horvitz 1985). To identify putative transposon-insertion alleles, we used this approach to isolate spontaneous mutants arising from the TR679 mutator strain (Collins et al. 1987). We isolated 17 mutants and then showed that these mutations failed to complement one of the following pre-existing mutations: lin-2(e1309), lin-10(e1439), lin-12(n950), lin-15(n309) and lin-31(n301). Thus, these mutations define new alleles of five previously identified genes: lin-10(n1299, n1387) I, lin-31(n1282, n1289, n1290, n1291, n1292, n1293, n1294, n1300, n1301) II, lin-12(n1295) III, lin-2(n1297, n1298) X, and lin-15(n1296, n1331, n1334) X.

To reduce the endogenous $\mathrm{Tcl}$ copy number to a number in which individual bands could be seen on a Southern blot probed with Tcl, the original mutator-derived isolate of lin-10(n1299) was crossed with a Bristol strain, which has a low number of Tcl copies. lin-10(n1299) males were mated with dpy-5 unc-13 hermaphrodites (dpy-5 and unc-13 are closely linked to lin-10). Cross progeny males, of genotype lin-10(n1299)/dpy-5 unc-13, were mated with $d p y-5$ unc- 13 hermaphrodites. Phenotypically wild-type cross progeny males from this mating are probably heterozygous for $\operatorname{lin}-10$ (n1299) but are reduced in Tcl copy number by approximately a factor of two. These males were mated with dpy-5 unc-13 hermaphrodites to reduce the Tcl copy number by another factor of two. After repeating this procedure eight times, we picked lin-10(n1299) homozygous animals, yielding a congenic strain derived mostly from Bristol except in the vicinity of lin-10(n1299).

To obtain revertants, non-vulvaless animals were isolated from the original strain carrying the lin-10(n1299) mutation. This original strain still carries the mut-2(r453) mutation. One revertant carried a mutation, $n 1329$, with a recessive suppressing phenotype linked to the $\mathrm{X}$ chromosome, indicating that $n 1329$ is an extragenic suppressor (S. Kim, unpubl.). A second revertant carried a mutation, n1326, with a dominant suppressing phenotype tightly linked to lin-10, consistent with its being an intragenic revertant. To show linkage to lin-10, n1299 n1326 revertant males were mated with dpy-5 hermaphrodites, yielding phenotypically wild-type cross progeny.
Of $\sim 8000$ animals screened, these progeny never segregated Vul animals, indicating that $n 1326$ is closely linked to lin $-10(P<2.2$ map units).

\section{Germ line transformation experiments}

Germ line transformation was performed essentially as described by Fire [1986\}, with the following minor modifications. Lucifer yellow was omitted from the injection solution, and the animals were not illuminated with ultraviolet light. Oocytes were injected in each of the proximal gonadal arms, aiming for a cytoplasmic location near the nucleus. About 10 oocytes from each animal were injected, using a cosmid DNA concentration of $\sim 100 \mu \mathrm{g} / \mathrm{ml}$. The DNA was prepared by two rounds of equilibrium density gradient centrifugation through cesium chloride gradients. Typically, $\sim 10$ non-Unc transformants were obtained after injecting 30 animals (most transformants were derived from different parents). None of the transformants in this study is likely to have cosmid sequences integrated into the host genome, as none of the transformants ever gave rise to a homozygous animal that transmitted the non-Unc phenotype to all of its progeny. Each of the transformants in this study transmits the non-Unc phenotype to some of its progeny (ranging from $10 \%$ to $90 \%$ ). Thus, the transforming DNA is genetically unstable, suggesting that it may be carried as an extrachromosomal array, as has been reported previously (Stinchcomb et al. 1985; Fire 1986; Way and Chalfie 1988).

Four lin-10 cosmids were used in these transformation studies (B234, AAE8, K7E6, and ZK265). Each of these cosmids contains all of the HindIII sites shown in Figure 5, and thus is likely to span the lin-10 locus. Southern blot experiments indicate that the sequences encoding the $14-\mathrm{kb}$ gene do not appear to have undergone significant deletion or rearrangement in these cosmids (data not shown).

\section{Molecular cloning and DNA sequence analysis}

Standard techniques were used for cloning and sequence analysis (Maniatis et al. 1982). To clone the $5.3-\mathrm{kb}$ EcoRI fragment containing $n 1299:: T c 1$, EcoRI-digested n1299 DNA was purified from an agarose gel fragment, cloned into $\lambda$ gt 10 and the resulting clones were screened by hybridization with a Tcl probe (pTc1). The insert was recloned into Bluescribe $\mathrm{Ml}^{+}$, and the $1.6-\mathrm{kb} \mathrm{Tcl}$ fragment was deleted by digesting with EcoRV (EcoRV cuts near both ends of Tcl) and religating the plasmid, yielding plin $10 \Delta \mathrm{A}$. A 1.8 -kb PvuII fragment derived from plin $10 \Delta \mathrm{A}$ was used as a probe to screen an ordered set of C. elegans genomic cosmid clones (a gift of G. Benian) and yielded genomic lin-10 cosmids AAE8 and BBDl. The 3.7-kb EcoRI band containing the $n 1299:: T c 1$ integration site was subcloned from AAE8, giving rise to plasmid p3.7.

These cosmids were analyzed by A. Coulson and J. Sulston, who are completing a physical map of the C. elegans genome $1>95 \%$ of the genome is represented by overlapping sets of cosmids; Coulson et al. 1986, 1988), and were found to overlap a previously identified set of cosmids that spans $\sim 4800 \mathrm{~kb}$ around lin-10 on chromosome I (A. Coulson and J. Sulston, pers. comm.). This set contains cosmids B234 and K7E6, both of which contain the 3.7-kb EcoRI fragment that is the site of in tegration of $n 1299:: T c 1$. Thus, cosmids AAE8, BBD1, B234, and K7E6 are candidates for containing the complete lin-10 gene.

Plasmid p3.7 was used as a probe to screen two existing $C$. elegans cDNA libraries constructed from poly $(\mathrm{A}){ }^{+} \mathrm{RNA}$ from mixed stage hermaphrodites (B. Meyer, J. Ahringer, unpubl.), yielding one clone from the first library and eight clones from 
the second. None of these clones was longer than $1 \mathrm{~kb}$, and most of the clones from the Ahringer library were prematurely terminated at the two internal EcoRI sites contained in the lin-10 cDNA sequence. We constructed a new C. elegans cDNA library in $\lambda$ gt 10 from poly $(A)+$ RNA from mixed stage hermaphrodites (S. Kim, unpubl.). Fifteen lin-10 cDNA clones were obtained by screening 250,000 clones from this library, as above, including c10-6, c10-7, c10-12, and c10-13. The 23 cDNA clones mentioned above hybridize to each other and have identical restriction maps over their regions of overlap.

Two cDNA clones (c10-4 and $\mathrm{c} 10-9)$ that do not share sequences with the 23 lin-10 cDNA clones were isolated by hybridization with the genomic clone, p3.7. Initially, we considered the possibility that these two cDNA clones correspond to a second gene, located completely within the intron of the $14-\mathrm{kb}$ lin-10 gene. However, this possibility is unlikely to be true, because sequence analysis shows that these clones have a high AT content and no long open reading frame, characteristics of noncoding $C$. elegans sequences (data not shown). These two clones were isolated only because they hybridize to a repeat within $\mathrm{p} 3.7$, indicating that they do not necessarily derive from this region but may derive from copies of this repeat in other regions of the genome.

Four lin-10 cDNA clones (c10-6, cl0-7, c10-12, and c10-13) were sequenced. Nested deletions were constructed by the method of Henikoff (1984), and double-stranded DNA was sequenced by the method of Sanger et al. (1977). c10-6, c10-12, and c10-13 end within several base pairs of each other at their $5^{\prime}$ ends. c10-13 contains a 9-bp deletion relative to the other two clones; otherwise, these three clones are identical in sequence for the region that they overlap.

No significant sequence similarity was found when the lin-10 sequence was compared to other sequences in the $\mathrm{Na}$ tional Biomedical Research Foundation Protein Sequence Database (Georgetown University Medical Center), the GenBank genetic sequence data bank, or the EMBL nucleotide sequence data library, using the FASTP algorithm.

c10-12 hybridizes to three regions of genomic DNA spanning $14 \mathrm{~kb}$ (S. Kim, unpubl.). The size of each exon in the hybridizing region was determined using RNase protection assays (Scherer 1986). A single-stranded RNA probe was synthesized from a cDNA template and hybridized to cosmid genomic clones. Unhybridized RNA was digested with RNase A, and the remaining probe was analyzed on a polyacrylamide gel. The sizes of the protected fragments indicate the sizes of the exons; the 5', middle and $3^{\prime}$ exons are $\sim 380 \mathrm{bp}, 600 \mathrm{bp}$, and $530 \mathrm{bp}$ in size, respectively. The sizes of these three putative exons total $\sim 1510 \mathrm{bp}$, consistent with the size of the cDNA clones.

\section{RNase protection assays}

To prepare staged RNA, worms were synchronized at different developmental stages (Meyer and Casson 1986). Worms were grown in S medium, and eggs were prepared by treating the entire population with $1 \%$ sodium hypochlorite and $0.5 \mathrm{M}$ sodium hydroxide in M9 media for $5 \mathrm{~min}$. The eggs were allowed to hatch in sterile M9, so that the starving larvae would all arrest in the middle of the first larval stage. The worms were fed after one day, and animals synchronized in different developmental stages were obtained by harvesting the culture at various times. We do not know whether starvation affects lin-10 RNA levels.

RNA was prepared by the method of Chirgwin et al. (1979), except that genomic DNA was sheared by sonication in the initial homogenate. For each experiment, $20 \mu \mathrm{g}$ of total RNA was used. A 420-bp fragment from a lin-10 cDNA clone, c10-7, was used to synthesize the antisense RNA probe used in Figures $4 \mathrm{C}$ and $8 \mathrm{~A}, \mathrm{~B}$. This fragment corresponds to bases $681-1100$ in the sequence in Figure 7. The fragment was cloned into Bluescribe $\mathrm{Ml3}^{+}$(Stratagene), made linear by cutting at a unique HindIII site in the vector, and transcribed with T7 RNA polymerase using $100 \mu \mathrm{Ci}$ of $\left.{ }^{32} \mathrm{P}\right] \mathrm{CTP}$ (Amersham, $800 \mathrm{Ci} / \mathrm{mmole}$ ) diluted with cold CTP to a final concentration of $40 \mu \mathrm{M}$. The act-1 V probe used in the experiments shown in Fig. 8A, and B was made by recloning pW-16-210 (Krause and Hirsh 1984) into Bluescribe $\mathrm{M}^{3} 3^{+}$. An RNA probe was synthesized using T3 polymerase with $20 \mu \mathrm{Ci}$ of $\left[{ }^{32} \mathrm{P} \mid \mathrm{CTP}\right.$ diluted with CTP to a final concentration of $400 \mu \mathrm{M}$. Thus, the act-1 probe was synthesized at one-fiftieth the specific activity of the lin-10 probe to compensate for the different abundances of these two RNAs. RNase protection experiments were performed as described (Zinn et al. 1983; Myers et al. 1985; Winter et al. 1985).

\section{Acknowledgments}

We thank Gian Garriga, Michael Stern, and Scott Clark for providing us with new lin-10 alleles; Mike Finney for providing pTcl plasmid; Guy Benian for providing his cosmid library; Barbara Meyer and Julie Ahringer for providing cDNA libraries; Alan Coulson and John Sulston for the identification of overlapping lin-10 cosmids; Maria Gallegos for help with the illustrations; and Burton Smith for providing the microinjection cap. We also thank Mike Herman, Anne Villeneuve, Scott Clark, Greg Beitel, Junying Yuan, and all of our other C. elegans colleagues at MIT for helpful advice and discussions. We acknowledge Anne Villeneuve, Bob Klein, Scott Clark, Chip Ferguson, and Cori Bargmann for their critical reading of this manuscript. S.K. was a fellow of the Helen Hay Whitney Foundation and is a Lucille P. Markey Scholar in Biomedical Science. This work was supported by research grants GM-24663 and GM-24943 from the U.S. Public Health Service. H.R.H. is an investigator of the Howard Hughes Medical Institute.

\section{Note added in proof}

Sequence data described in this paper have been submitted to the EMBL/GenBank Data Libraries under accession number $\mathrm{X} 51321$.

\section{References}

Amrein, H., M. Gorman, and R. Nothiger. 1988. The sex-determining gene tra-2 of Drosophila encodes a putative RNA binding protein. Cell 55: 1025-1035.

Banerjee, U., P.J. Renfranz, D.R. Hinton, B.A. Rabin, and S. Benzer. 1987. The sevenless ${ }^{+}$protein is expressed apically in cell membranes of developing Drosophila retina; it is not restricted to cell R7. Cell 51: 151-158.

Breeden, L. and K. Nasmyth. 1987. Similarity between cellcycle genes of budding yeast and fission yeast and the Notch gene of Drosophila. Nature 329: 651-654.

Brenner, S. 1974. The genetics of Caenorhabditis elegans. Genetics 77: 71-94.

Chirgwin, J., A. Przybyla, R. MacDonald, and W. Rutter 1979. Isolation of biologically active ribonucleic acid from sources enriched in ribonucleases. Biochemistry 18: 5295-5299.

Collins, J., E. Forbes, and P. Anderson. 1989. The Tc3 family of transposable genetic elements in Caenorhabditis elegans. Genetics 121: 47-55.

Collins, J., B. Saari, and P. Anderson. 1987. Activation of a transposable element in the germ line but not the soma of $C$. elegans. Nature 328: 726-728. 
Coulson, A., J. Sulston, S. Brenner, and J. Karn. 1986. Toward a physical map of the Caenorhabditis elegans genome. Proc. Nat. Acad. Sci. 83: 7821-7825.

Coulson, A., R. Waterston, J. Kiff, J. Sulston, and Y. Kohara. 1988. Genome linking with artificial chromosomes. Nature 335: $184-186$.

Eide, D. and P. Anderson. 1988. Insertion and excision of $C$. elegans transposable element Tc1. Mol. Cell. Bio. 8: 737746.

Emmons, S.W., L. Yesner, K.-S. Ruan, and D. Katzenberg. 1983. Evidence for a transposon in C. elegans. Cell 32: 55-65.

Ferguson, E.L. and H.R. Horvitz. 1985. Identification and characterization of 22 genes that affect the vulval cell lineages of the nematode Caenorhabditis elegans. Genetics 110: 1772 .

- 1989. The multivulva phenotype of certain Caenorhabditis elegans mutants results from defects in two functionally redundant pathways. Genetics 123: 109-121.

Ferguson, E.L., P.W. Sternberg, and H.R. Horvitz. 1987. A genetic pathway for the specification of the vulval cell lineages in Caenorhabditis elegans. Nature 326: 259-267.

Finney, M., G. Ruvkun, and H.R. Horvitz. 1988. The C. elegans cell lineage and differentiation gene unc- 86 encodes a protein with a homeodomain and extended similarity to transcription factors. Cell 55: 757-769.

Fire, A. 1986. Integrative transformation of Caenorhabditis elegans. EMBO J. 5: 2673-2681.

Goralski, T. J., J.-E. Edstrom, and B.S. Baker. 1989. The sex determination locus transformer-2 of Drosophila encodes a polypeptide with similarity to RNA binding proteins. Cell 56: $1011-1018$.

Greenwald, I.S., 1985. lin-12, a nematode homeotic gene, is homologous to a set of mammalian proteins that includes epidermal growth factor. Cell 43: 593-590.

Greenwald, I.S. and H.R. Horvitz 1980. unc-93(e1500): A behavioral mutant of Caenorhabditis elegans that defines a gene with a wild-type null phenotype. Genetics 96: 147164.

Greenwald, I.S., P.W. Sternberg, and H.R. Horvitz. 1983. The lin-12 locus specifies cell fates in Caenorhabditis elegans. Cell 34: 435-444.

Grosveld, F., G.B. van Arsendelft, D.R. Greaves, and G. Kolias. 1987. Position independent high level expression of the human $\beta$-globin gene in transgenic mice. Cell 51: 975-985.

Hafen, E., K. Basler, J.E. Edstroem, and G.M. Rubin. 1987. sevenless, a cell-specific homeotic gene of Drosophila, encodes a putative transmembrane receptor with a tyrosine kinase domain. Science 236: 55-63.

Harris, W. A., W.S. Stark, and J.A. Walker. 1976. Genetic dissection of the photoreceptor system in the compound eye of Drosophila melanogaster. J. Physiol. 256: 415-439.

Henikoff, S. 1984. Unidirectional digestion with exonuclease III in DNA sequence analysis. Gene 28: 351-359.

Herman, R.K. 1988. Genetics. In The nematode Caenorhabditis elegans. (ed. W. Wood), pp. 17-46. Cold Spring Harbor Laboratory Press, Cold Spring Harbor, New York.

Hodgkin, J., M. Edgley, D.L. Riddle, and D.G. Albertson. 1988. Appendix 4: Genetics. In The Nematode Caenorhabditis elegans. (ed. W. Wood), pp. 491-586. Cold Spring Harbor Laboratory Press, Cold Spring Harbor, New York.

Horvitz, H.R. and J.E. Sulston. 1980. Isolation and genetic characterization of cell lineage mutants of the nematode Caenorhabditis elegans. Genetics 96: 435-454.

Kiff, J.E., D.G. Moerman, L.A. Schriefer, and R. Waterston. 1988. Transposon-induced deletions in unc-22 of C. elegans associated with almost normal gene activity. Nature 331: $631-633$.
Kimble, J., 1981. Alterations in cell lineage following laser ablation of cells in the somatic gonad of Caenorhabditis elegans. Dev. Biol. 87: 286-300.

Kimble, J. and D. Hirsh. 1979. The post-embryonic cell lineages of the hermaphrodite and male gonads in Caenorhabditis elegans. Dev. Biol. 70: 396-417.

Krause, M. and D. Hirsh. 1984. Actin gene expression in Caenorhabditis elegans. In Molecular biology of the cytoskeleton, (ed. G.G. Borisy, D.W. Cleveland, and D.B. Murphy), pp. 244-258. Cold Spring Harbor Laboratory Press, Cold Spring Harbor, New York.

Laski, F.A., D.C. Rio, and G.M. Rubin. 1986. Tissue specificity of Drosophila P element transposition is regulated at the level of mRNA splicing. Cell 44: 7-19.

Lipshitz, H.D., D.A. Peattie, and D.S. Hogness. 1987. Novel transcripts from the Ultrabithorax domain of the bithorax complex. Genes Dev. 1: 307-322.

Maniatis, T., E.F. Fritsch, and J. Sambrook. 1982. Molecular cloning: A laboratory manual. Cold Spring Harbor Laboratory Press, Cold Spring Harbor, New York.

Meneely, P.M. and R.K. Herman. 1979. Lethals, steriles and deficiencies in a region of the $\mathrm{X}$ chromosome of Caenorhabditis elegans. Genetics 92: 99-115.

Meyer, B.J. and L.P. Casson. 1986. Caenorhabditis elegans compensates for the difference in $\mathrm{X}$ chromosome dosage between sexes by regulating transcription levels. Cell 47: $871-881$.

Moerman, D.G., G.M. Benian, and R.H. Waterston. 1986. Molecular cloning of the muscle gene unc-22 in C. elegans by Tcl transposon tagging. Proc. Nat. Acad. Sci. 83: 25792583.

Myers, R.M., Z. Larin, and T. Maniatis. 1985. Detection of single base substitutions by ribonuclease cleavage at mismatches in RNA : DNA duplexes. Science 230: 1242-1246.

Park, E. and H.R. Horvitz. 1986. Mutations with dominant effects on the behavior and morphology of the nematode Caenorhabditis elegans. Genetics 113: 821-852.

Peifer, M., F. Karch, and W. Bender. 1987. The Bithorax Complex: Control of segmental identity. Genes Dev. 1: 891-898.

Rosenquist, T.A. and J. Kimble. 1988. Molecular cloning and transcript analysis for $\mathrm{fem}-3$, a sex-determination gene in Caenorhabditis elegans. Genes Dev. 2: 606-616.

Sanger, F., S. Nicklen, and A. Coulson. 1977. DNA sequencing with chain-terminating inhibitors. Proc. Nat. Acad. Sci. 74: 5463-5467.

Scherer, S., 1986. Regulated yeast promoters produced by DNA rearrangements in vivo. J. Mol. Bio. 191: 355-365.

Seydoux, G. and I. Greenwald. 1989. Cell autonomy of the lin-12 function in a cell fate decision in C. elegans. Cell 57: $1237-1245$.

Sternberg, P.W. 1988. Lateral inhibition during vulval induction in C. elegans. Nature 335: 551-554.

Sternberg, P.W. and H.R. Horvitz. 1984. The genetic control of cell lineage during nematode development. Annu. Rev. Genet. 18: 489-524.

1986. Pattern formation during vulval development in Caenorhabditis elegans. Cell 44: 761-772.

. 1989. The combined action of two intercellular signalling pathways specifies three cell fates during vulval induction in C. elegans. Cell 58: 679-693.

Stinchcomb, D., J. Shaw, S. Carr, and D. Hirsh. 1985. Extrachromosomal DNA transformation of C. elegans. Mol. Cell Biol. 5: 3484-3496.

Sulston, J.E. and S. Brenner. 1974. The DNA of Caenorhabditis elegans. Genetics 77: 95-104.

Sulston, J.E. and H.R. Horvitz. 1977. Postembryonic cell lineages of the nematode Caenorhabditis elegans. Dev. Biol. 
56: $110-156$.

1981. Abnormal cell lineages in mutants of the nematode Caenorhabditis elegans. Dev. Biol. 82: 41-55.

Sulston, J.E. and J.G. White. 1980. Regulation and cell autonomy during postembryonic development of Caenorhabditis elegans. Dev. Biol. 100: 64-119.

Suzuki, D.T. 1970. Temperature-sensitive mutations in Drosophila melanogaster. Science 170: 695-706.

Tomlinson, A., D.D.L. Bowtell, E. Hafen, and G. Rubin. 1987. Localization of the sevenless protein, a putative receptor for positional information, in the eye imaginal disc of Drosophila. Cell 51: 143-150.

Way, J.C. and M. Chalfie. 1988. mec-3, a homeobox-containing gene that specifies differentiation of the touch receptor neurons in C. elegans. Cell 54: 5-16.

Wills, N., R.F. Gesteland, J. Karn, L. Barnett, S. Bolten, and R.H. Waterston. 1983. The genes sup - $7 X$ and sup -5 III of Caenorhabditis elegans suppress amber nonsense mutations via altered transfer RNA. Cell 33: 575-583.

Winter, E., F. Yamamoto, C. Almoguera, and M. Perucho. 1985. A method to detect and characterize point mutations in transcribed genes: Amplification and overexpression of the mutant c-Ki-ras allele in human tumor cells. Proc. Nat. Acad. Sci. 82: 7575-7579.

Yochem, J., K. Weston, and I. Greenwald. 1988. The Caenorhabditis elegans lin-12 gene encodes a transmembrane protein with overall similarity to Drosophila Notch. Nature 355: $547-550$.

Zinn, K., D. Dimaio, and T. Maniatis. 1983. Identification of two distinct regulatory regions adjacent to the human $\beta$-interferon gene. Cell 34: 865-879. 


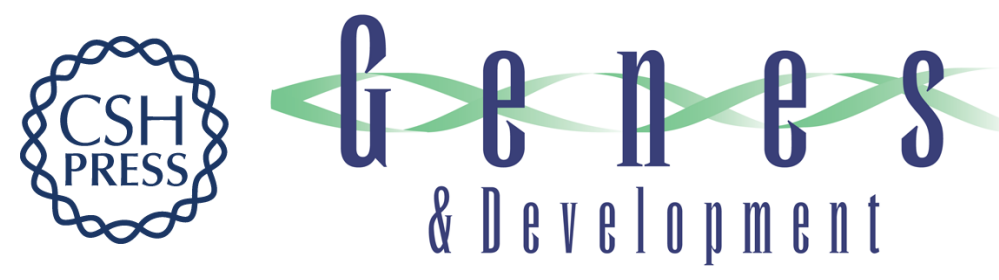

\section{The Caenorhabditis elegans gene lin-10 is broadly expressed while required specifically for the determination of vulval cell fates.}

S K Kim and H R Horvitz

Genes Dev. 1990, 4:

Access the most recent version at doi:10.1101/gad.4.3.357

References This article cites 56 articles, 21 of which can be accessed free at:

http://genesdev.cshlp.org/content/4/3/357.full.html\#ref-list-1

License

Email Alerting

Service

Receive free email alerts when new articles cite this article - sign up in the box at the top right corner of the article or click here.

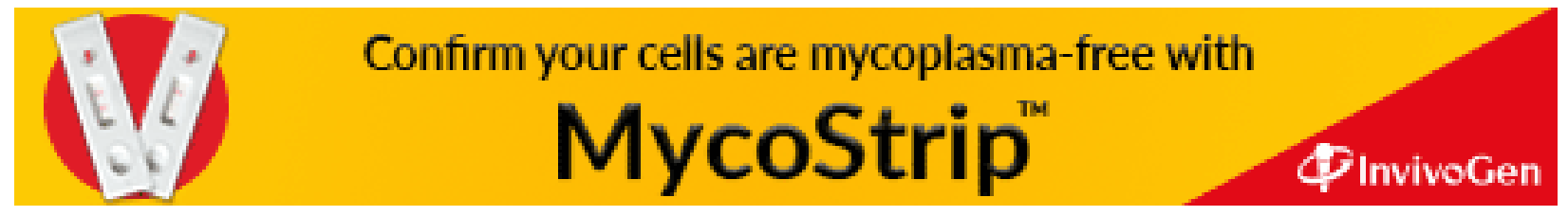

